\title{
LA-8307-MS
}

Informal Report
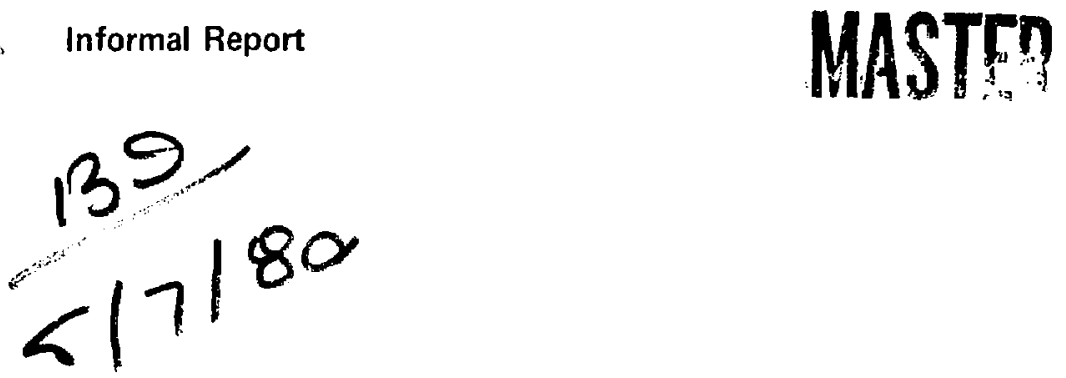

\section{LAMPF Beam Line Polarimeters}

$\frac{\sigma}{\frac{\sigma}{0}}$

吅

$\frac{2}{\frac{1}{0}}$ 
LA-8307-MS

Informal Report

UC-28

Issued: April 1980

\section{LAMPF Beam Line Polarimeters}

Michael W. McNaughton
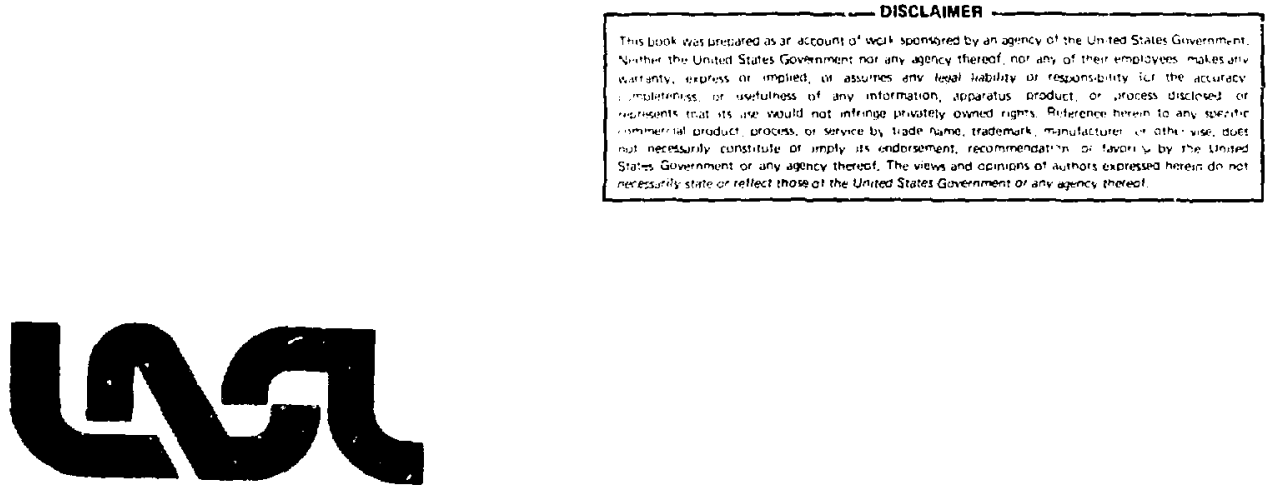


\section{LAMPF BEAM LINE POLARIMETERS}

by

Michael W. McNaughton

\section{ABSTRACT}

Polarimeters have been built to measure the polarization of the LAMPF beam (400 to $800 \mathrm{MeV}$ ). The polarimeters are simple and reliable and give a $1 \%$ result ( 1 imited by counting statistics) within a few minutes. The design, calibration, and use of these devices are described. 


\section{LAMPF BEAM LINE POLARIMETERS}

by

Michael W. McNaughton

\section{INTRODUCTION}

This report describes the beam line polarimeters that are used in the primary $\mathrm{P}^{-}$beams at LAMPF to monitor and measure the polarization of the beam. The devices are secondary standards of polarization and are calibrated primarily by the ion source quench ratio technique (Section VII). Some independent checks of this technique are discussed in Section VIII and IX.

\section{SCATTERING EXPERIMENTS}

The polarization $\mathrm{P}$ of a beam of nucleons is given by

$$
P=\langle\sigma\rangle=(\uparrow-\uparrow) /(\uparrow+\uparrow) \text {, }
$$

where $\uparrow,+$ denote number of part,cles with spin up,down. If a polarized beam, $P>0$, scatters from a nucleus then more particles scatter left $(L)$ than right $(R)$, giving an asymmetry

$$
e=(L-R) /(L+R) \text {. }
$$

The analyzing power $A$ is defined by

$$
\mathrm{e}=\mathrm{AP} \text {. }
$$

The subscript $n$ or $y$ is often added to indicate that the polarization direction is perpendicular to the scattering plane; we shall be concerned with no other case, and will assume that the other cases, $A_{x}=A_{z}=0$ (by parity conservation). The term "polarization" is sometimes used to refer to the polarization produced in a recoil beam by scattering an unpolarized beam from a nucleus. since this quantity is equal to the analizing power (by time reversal) the symbol 
A will be used for the polarization (or analyzing power) of a reaction, and $\mathrm{P}$ for the polarization of a beam.

A beam polarized up and scattered right is equivalent to a beam polarized down and scattered left, so that the yields of two detectors (1eft and right) may be replaced by the yields in one detector for beam spin up and down. If we combine these two techniques and scatter both left and right simultaneously while also periodically flipping the beam spin, we have four yields, $L t, L t, R t$, Rt. If we redefine

$$
L=\sqrt{L \uparrow R \downarrow} \quad \text { and } R=\sqrt{R \uparrow L \downarrow} \quad,
$$

then almost all experimental uncertainties and instrumental asymmetries cancel to a high order'. This technique has been used to measure asymmetries to \pm .002 , the error being determined solely by counting statistics ${ }^{2,17}$.

\section{THE BEAM LINE POLARIMETERS}

In order to obtain the analyzing power ( $A$, which contains the physics) from the experimentally observed asymmetry (e) it is necessary to know the beam polarization $P[E q .(3)]$. The beam line polarimeters are secondary standards of known analyzing power. A typical experiment measures the asymmetry for the reaction of interest relative to the asymmetry of the polarimeter.

The reaction chosen for the polarimeters is basically $p p \rightarrow p p$. The reasons for this choice are as follows:

A. Near $800 \mathrm{MeV}$ the analyzing power for $\mathrm{pp} \rightarrow \mathrm{pp}$ is the highest of any known reaction.

B. The broad maximum near $17^{\circ}$ lab makes the device insensitive to simall errors in angle.

C. The angle of this maximum remains almost unchanged over a wide range of energies, minimizing the need for geometrical changes to accommodate energy changes.

D. Detecting both final state protons at conjugate angles gives a very clean signal of elastic scattering.

E. $\mathrm{CH}_{2}$ makes a very convenient target.

The same reaction is used at TRIUMF ${ }^{3}$ and ZGS. The possible need to detect recoil protons at very low energy led TRIUMF to choose a reaction angle of $24^{\circ}$ 
lab. The large beam at the ZGS led to the detection of only one final state particle.

The LAMPF 1 ine EPB and LB polarimeters are shown in Figs. 1 and 2. Protons elastically scattered from $\mathrm{CH}_{2}$ are detected near $17^{\circ}$ and $67^{\circ}$ lab in each of four directions, left, right, up and down (the coincidence of $17^{\circ}$ left and $67^{\circ}$ right is known, by convention, as "Left"). The 1eft-right detectors measure the vertical $(y)$ component of beam polarization while the up-down detectors monitor the horizontal-transverse $(x)$ component. It is impossible to monitor the ?ongitudinal ( $z$ ) component directly.

The detectors in a11 cases are plastic scintillators mounted on LAMPF standard photomultipliers chosen to allow narrow time coincidence windows, and for simplicity and reliability. Energy deposited in the EPB and Line B detectors is about $3 \mathrm{MeV}$. The only essential differences between the beam 1 ine polarimeters are in geometry, necessitated by the different beam geometries in the different locations (see Table I).

The electronics consists in principle of HV power for the phototubes ( $\approx 1700 V)$, discriminators for the anode signals, and fast coincidences. Typical performance is illustrated in Figs. 3 and 4 . Each coincidence $(L, R, U, D)$ is scaled in two CAMAC scalers, one gated for "Normal" beam spin ( $N)$, the other for "Reverse" (R). The L-R and U-D asymmetries are calculated from the four numbers using the formalism of Section II [Eg̣s. (2), (4) and reference 2].

\section{SOURCES OF ERROR IN THE POLARIMETERS}

The EPB polarimeter has been shown to be reproducible to better than $0.5 \% ?, 17$ Some precautions regarding possible errors are in order, however.

For accurate work, a delayed coincidence ( $30 \mathrm{~ns}$ ) is included, recorded and subtracted. Random coincidences can be calculated approximately knowing that the singles rate in each detector is about 20 times the coincidence rate and that the resolving time is about $10 \mathrm{~ns}$ (see Fig. 4). Thus, the randoms rate (for 0.08 duty factor)

$$
R=\frac{(20 \times C)^{2}}{0.08} \times 10^{-8},
$$

where $C$ is the coincidence rate. If $C$ is $10^{3} \mathrm{~Hz}$ for example then $\mathrm{R}=50 \mathrm{~Hz}$. Randoms have an asjometry about half that of true coincidences. 
In the case of chopped beam, some care should be taken to ensure that randoms are correctly monitored. The delay is set to 30 ns to accommodate 1 in 16 chopped beam.

Norinally, data from the scattering experiment and the polarimeter are collected over exactly the same time period so that variations in the polarization of the beam are exactly compensated for. This might not be the case, however, if the beam polarization changes within the macropulse and dead times are such that the experiment and the polarimeter on average sample different parts of the macropulse. It is conceivable that the beam polarization (which is a function of phase space ${ }^{4,5}$ ) could differ between the polarimeter and the experimental target especially if one target does not intercept all of the beam. A 4\% difference has been observed at LAMPF between LB and EPB ${ }^{5}$; corisequently, comparison between different beam 1 ines should be made with caution.

It should be noted that the magnitude of the beam polarization need not be the same for beam spin Normal and Reverse. (With slow spin reversal this is typically a result of a drift in the tune of the spin filter. With rapid reversal a few percent depolarization of the adiabatically reversed state is inevitable.) It is very difficult to distinguish such a difference between polarizations and an instrumental asymmetry in the polarimeter. For example, yields of $L_{\uparrow}=R_{t}=$ 150, $L \downarrow=R \uparrow=50$ would indicate $e=0.5$. A different set of readings $L \uparrow=155$, $R_{t}=145, L \downarrow=55, R \uparrow=45$ could indicate either a larger polarization for $\uparrow$ than + or an instrumental asymmetry favoring the left detector (e.g., beam steered left). Data with unpolarized beam can be used to investigate the instrumental asymmetry and hence resolve this ambiguity.

A full two-arm experiment (scattering left and right simultaneously) is insensitive to this ambiguity, since writing the beam polarizations as $(P+\delta)$ and $-(P-i)$ and taking the geometric means [Eq. (4)] gives (to first order)

$$
e=A P\left(1+A^{2} \delta^{2} / 2\right) .
$$

In a single-arm experiment (or if cross sections are to be extracted from polarized beam data), however, such differences may be significant.

$$
\mathrm{e}=\frac{A P}{1+A \delta} \text {. }
$$

Events are detected not only from the reaction $p p \rightarrow p p$ but also from $c(p, 2 p)$ (quasi-free scattering). The quasi-free events from the carbon in $\mathrm{CH}_{2}$ are about equal in number to those from the two hydrogen nuclei, but the angular correlation 
is spread over about $10^{\circ}$ by the Fermi motion in the nucleus. Consequently, about $5 \%$ of the prompt events are from carbon, depending on the geometry. Since these events have about half the asymmetry $(A=.275 \pm .015$ at $800 \mathrm{MeV})$ this leads to an effective analyzing power for each polarimeter that is a few percent lower than for $p p \rightarrow p p$ (differing for each polarimeter, e.g., $3 \%$ for EPB at $800 \mathrm{MeV}$ ).

This correction could change, however, as a result of the following circumstances:

A. Substantial misalignment or unusually large beam spots would reduce the combined solid angle of primary and conjugate detectors for $\mathrm{pp} \rightarrow \mathrm{pp}$ but scarcely affect the solid angle for $c(p, 2 p)$. This is minimized by making the forward detectors define the sol id angle for typical beam spots (see Table I). (See also note 1 of Table 1.)

B. Substantial depletion of hydrogen from the $\mathrm{CH}_{2}$ would also increase the ratio of $\mathrm{C} / \mathrm{H}$ events. (Reference 5 estimates . $1 \% / \mathrm{nA}-\mathrm{hr} \mathrm{H}$ depletion $(=.1 \% \times .03$ change in $A$ for EPB polarimeter $)$ for a $1 \mathrm{~mm}$ radius beam spot).

$\$$ dependence can enter in two ways, both of which are normally negligible. Taking the simplest case, namely, vertical polarization, if the U-D asymmetry is non-zero then the polarization vector is tilted from the vertical $(y)$. (There may, of course, be a $z$ component, as well as the $x$ component sensed by the U-D asymmetry.) If both the main experiment and the L-R polarimeter asymmetry are sensitive only to the vertical ( $y$ ) component, then the possible existence of $x$ or $z$ components requires no correction. An important exception, however, is a quench ratio experiment where the quench ratio measures the length of the polarization vector, while the L-R polarimeter asymmetry measures only the $y$ component.

The second $\phi$ dependence concerns the average value of $\cos \phi$ over the $\phi$ acceptance of the detectors. For the EPB and LB polarimeters, the $\phi$ acceptance is about $7^{\circ}$ (Table I), for which $\langle\cos \phi\rangle=.999$, a negligible correction. The $\phi$ acceptance of the main experiment may, however, be significant at forward angles.

\section{THE RECOIL PROTON POLARIMETER}

The recoil proton polarimeter (which may also be used for neutrons) is designed for double- and triple-scattering experiments. (A triple-scattering experiment is the same as a double-scattering experiment, but using polarized 
beam; historically an extra scattering was required to produce the polarized beam.) The device is similar to those at TRIUMF ${ }^{7}, \operatorname{SIN}^{8}$, and $A N L^{9}$, and cons is ts of MWPCS to define the particles before the (second) target, and MWPCs to define a charged particle after the target ${ }^{21}$.

The standard target is carbon (up to $25 \mathrm{~cm}$ thick) with the reaction being $c(p, z)$ (z-charged). Though this target lacks the advantages listed in Section II for $\mathrm{CH}_{2}$, it has the essential advantage of high cross section. The analyzing power is about half that of hydrogen near $800 \mathrm{MeV}$, but twice that of hydrogen at lower energies (few hundred MeV). Carbon was historically chosen because of the possibility of separating elastic from inelastic events at lower energies. The SIN device selects nearly elastic events by range or $\mathrm{dE} / \mathrm{dX}$ selection, with a gain of about $20 \%$ in analyzing power 8 . No such selection is attempted at TRIUMF or A.NL (the difficulty of such selection increases rapidly at higher energies). Carbon is retained as the standard target because of the advantages of cross calibration with previous work. There are indications, however, that comparatie analyzing powers may be obtained from a variety of targets 10,11 .

The device is mentioned here because of its potential use for doublescattering calibrations, which would be an independent check of the quench ratio calibration.

VI. DOUBLE-SCATTERING CALIBRATIONS

Double-scattering calibrations of the polarimeters have been discussed in Ref. 12 and 13 but not yet performed.

\section{QUENCH RATIO CALIBRATIONS}

The Lamb-shift ion source at LAMPF possesses the capability, unique above Van de Graaff energies, of measuring the beam polarization from the atomic physics properties of the source. This technique, known as the "quench ratio," was developed at the Los Alamos Van de Graaff accelerator ${ }^{4}$ where it has been used and cross checked against $p-{ }^{4} \mathrm{He}$ scattering ${ }^{14}$ to obtain a calibration to $\pm 0.4 \%$.

The method has been described in detail ${ }^{2}$ and so will only be described briefly here. The LAMPF ion source consists of three major components: cesium ce11, spin filter, and argon cell. The cesium cell provides a beam rich in hydrogen atoms in the $2 \mathrm{~s}$ excited state. The spin filter "quenches" to the ground state all but those in one electron-proton spin configuration. The argon cell 
preferentially ionizes the 2s-state atoms, giving a beam consisting primarily of polarized particles from the $2 \mathrm{~s}$ state, but with some unpolarized background from ionization of ground-state atoms.

When the spin filter is detuned, all $2 \mathrm{~s}$ atoms are quenched so that only this unpolarized background remains. The ratio of beam intensities for these two cases, known as the "quench ratio $Q$ ", yields the beam polarization $P=1-1 / Q+P_{q} / Q$. [Since $P_{q}$ is typically -0.03 , the term $P{ }_{q} / Q$ is a small (0.3\%) correction for the slight polarization $\mathrm{P}_{\mathrm{q}}$ of the quenched beam.] Figure 5 shows the effect on both polarization and intensity of detuning the spin filter by changing the magnetic field $B$ (known as a " $\Delta B$ quench").

The major assumptions behind this measurement of the beam polarization are as follows:

A. The unpolarized component must be unperturbed by the quenching. We have compared the $\Delta B$ quench with a $\Delta E$ quench obtained by changing the spin filter electric field, expecting any perturbation to be different in these two cases. Comparison of the two results indicates a $0.2 \%$ to $0.5 \%$ correction in the $\Delta E$ case.

B. There must be no depolarization between spin filter and experimental target. The quench ratio measures the polarization as it existed in the spin filter. Subsequent depolarization would not affect the measured quench ratio and would therefore lead to error.

The possibility of depolarization is difficult to check empirically. A calculation was made in $1968^{15}$ which indicated that depolarization in the accelerator would be of the order of $0.1 \%$. Our empirical checks of depolarization have been limited to a comparison of different phase-space components measuring in each case the ratio of polarization measured by the quench ratio and by the EPB polarimeter. This "effective analyzing power" should not change. If depolarization occurs, we expect that different phase-space components of tine beam will be differently affected. Furthermore, since the entire $\mathrm{H}^{-}$beam spends much of $i$ ts time off axis in the accelerator, mannetic depolarizing effects should also precess the average spin direction, and should vary from one cycle to the next depending on the accelerator tune. These empirical checks so far indicate that depolarization is $0 \pm 1 / 2 \%$.

Within the ion source, we have observed up to $30 \%$ depolarization under extreme conditions (e.g., spin filter and argon cell fields opposed). With 
reasonable settings, we do not observe any change as a function of field, indicating $0 \pm 1 / 2 \%$ depolarization in the source.

It has been observed ${ }^{4,5}$ that the polarized and unpolarized components of the beam have different phase space so that phase-space selection by beam stripping can change the average polarization. It is therefore essential to measure the quench ratio at the scattering target. Unfortunately, we are then at the mercy of accelerator noise which causes intensity fluctuations unconnected with the quench ratio that we wish to measure. Averaging out the accelerator noise is time consuming and limits the accuracy that we have obtained at this time.

\section{POLARIZED TARGET CALIBRATION}

The pp analyzing power can be measured by scattering polarized beam from unpoiarized target or unpolarized beam from polarized target. By comparing these two results one can compare the calibrations of the polarized beam and the polarized target. Such a comparison was made using the data from the spin correlation experiment (Experiment 27) of $1977^{12,16}$. The results are consistent within experimental errors with the quench ratio calibrations 2,17 .

The polarized target calibration is entirely independent of the quench ratio calibration. During the scattering, the target material $\left(\mathrm{C}_{3} \mathrm{H}_{8} \mathrm{O}_{2}\right)$ is polarized (enhanced $P \approx 0.83$ ) by optical pumping in a 2.5 t magnetic field, while cooled to about $0.4 \mathrm{~K}$ in 7 iquid ${ }^{3} \mathrm{He}$. The polarization is proportional to the integrated NMR signal as a frequency modulated RF field passes through the proton resonance. The absolute calibration is obtained by comparing this signal with the "thermal equitibrium" signal obtained after the proton spin temperature has been allowed to equalize with the temperatures of the ${ }^{3} \mathrm{He}$ bath. The polarization is then known from Boltzman statistics, knowing the magnetic field and temperature $\left(\approx 1\right.$ Kelvin, from the ${ }^{3} \mathrm{He}$ vapor pressure).

Repeated measurements spanning several months were consistent to about $0.5 \%$. The average ratio of the independent calibrations from the quench ratio and the NMR was

$$
\frac{P(\text { quench })}{P(\text { NMR })}=1.002 \pm 0.005 \text {. }
$$

The \pm 0.005 error represents the internal consistency only. Overall systematic errors that might arise in the NMR calibration are discussed in Ref. 16, and are estimated to be no more than $\pm 2 \%$. 


\section{COMPARISON WITH OTHER LABORATORIES}

A calibration of the $\mathrm{P}^{-}$beam polarization at $428 \mathrm{MeV}$ was performed in $1978^{17}$ to compare with recent calibrations at TRIUMF ${ }^{18,19}$. The quench ratio technique was used to calibrate the EPB polarimeter at $428 \mathrm{MeV}$. Standard techniques were then used to measure the asymmetry in $p p \rightarrow p p$ at $24^{\circ} 1 \mathrm{ab}$. The LAlPF result $A_{y}=0.387 \pm .008$ at $428 \mathrm{MeV}$, compares reasonably with the TRIUMF calibrations of $0.381 \pm .006$ at $410 \mathrm{MeV}$ and $0.400 \pm .004$ at $440 \mathrm{ileV}^{18}$, and the phase shift fit, 0.385 at $425 \mathrm{MeV}^{20}$. The performance of the ion source during this period was, however, unusual. Of particular concern was the observed polarization of 0.18 for the quenched beam, which necessitated a $3 \%$ correction to the quench ratio calibration. Two calibrations at $796 \mathrm{MeV}$ performed at the same time were $0.6 \%$ and $1.5 \%$ lower than usual. Consequently, a correction factor of $1.01 \pm .02$ has been applied to the $428 \mathrm{MeV}$ data.

\section{ACKNOWLEDGMENTS}

I would like to thank my colleagues in MP-Division and of LAMPF Experiment 27 for their encouragement, advice and assistance in building, understanding and calibrating the beam line polarimeters. Of particular importance were Bob Macek, 01 in van Dyck, Earl Hoffman, Dick Werbeck, Ralph Stevens, Phil Chamberlin, Harvey Willard, Nick king and Phil Bevington. I would also like to thank Gloria Garcia for typing and assembling this report. 


\section{REFERENCES}

1. G. G. Ohisen and P. W. Keaton, Nucl. Instr. 109 (73) 41.

2. R. Bevington et al., Phys. Rev. Lett. 41 (78) 384.

3. C. Amsler et al., Nucl. Instr. 144 (77) 401.

4. G. G. Ohlsen, LASL report LA-4451 (1970).

5. M. W. Mcivaughton, MP-13 report, MP-13/MWM/A79-04 (1979).

6. H. B. Willard et al., Phys. Rev. C14 (1976) 1545.

7. G. Waters et a1., Nucl. Instr. 153 (1978) 401.

8. D. Aebisher et al., Nucl. Instr. 124 (1975) 49.

9. G. W. Abshire et al., Phys. Rev. D12 (1975) 3393.

10. M. G. Meshcheriakov et al., Sov. Phys. JETP4 (1957) 337.

11. J. E. Simmons, Phys. Rev. 104 (1956) 4 !6.

12. M. W. McNaughton, LAMPF Users Newsleter 10.1 (February 1978).

13. H. A. Thiessen (spokesperson), LAMPF Experiment 392 proposal (1978).

14. G. G. Ohlsen et al., Phys. Rev. Lett. 27 (1971) 559.

15. R. M. Mobley, report BNL 50120 (1968) 430.

16. M. W. McNaughton et al., "Measurement of the Spin Correlation Paraneter $A_{n n}$ in pp Elastic Scattering at $796 \mathrm{MeV}$," to be published.

17. M. W. McNaughton et al., "Polarization Analyzing Power $A(\theta)$ in pp Elastic Scattering at $796 \mathrm{MeV}$," to be published.

18. C. Amster et al., J. Phys. G4.7 (1978) 1047.

19. L. G. Greeniaus et al., TRIUMF preprint TRI UAE-5012 (1979). (Submitted to Nuclear Physics.)

20. D. V. Bugg et al., J. Phys. G4 (1978) 1025.

21. M. W. McNaughton, MP-13 internal report, MP-13/MWM/X80-01 (1980). 


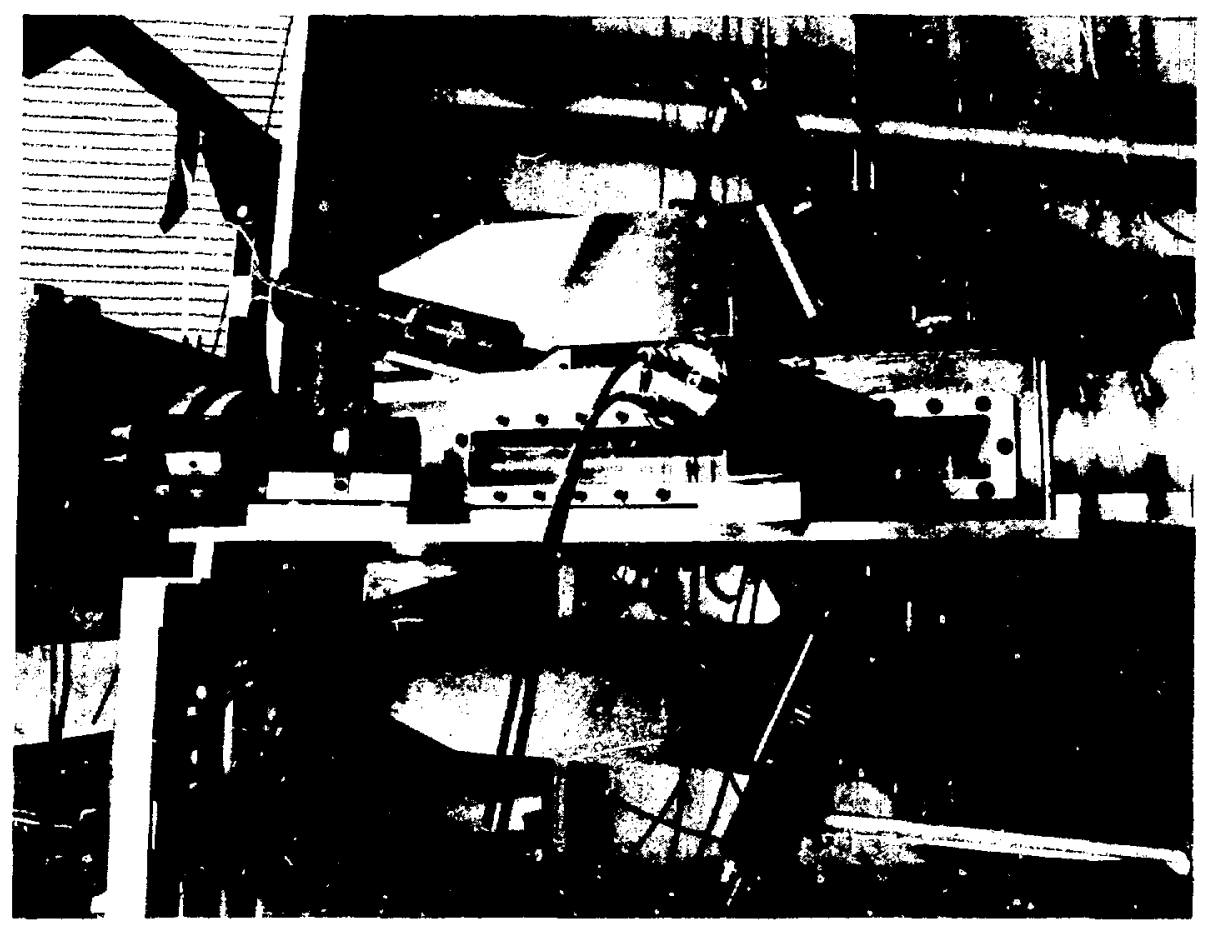

Fig. 1 - EPB polarimeter.

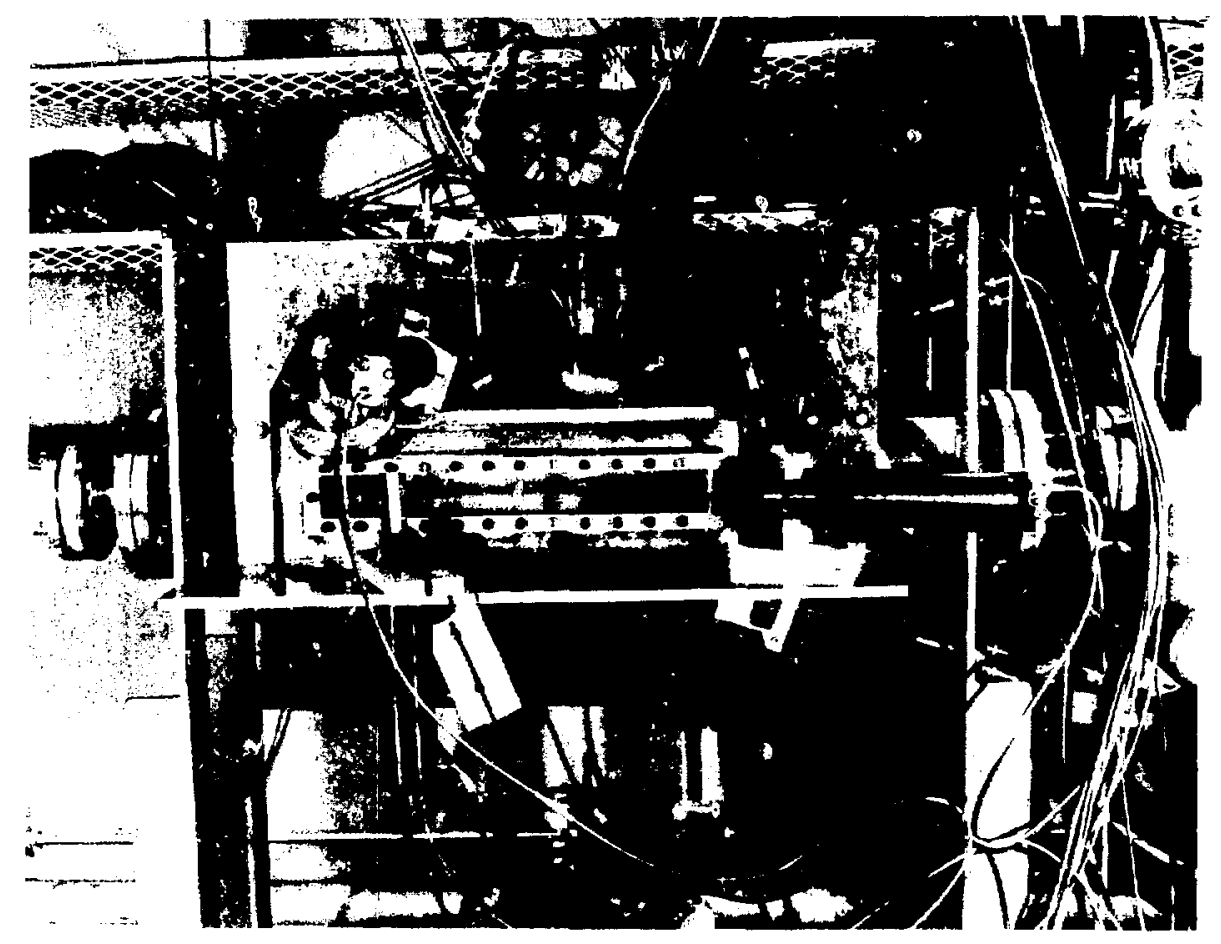

Fig. 2 - LB polarimeter. 

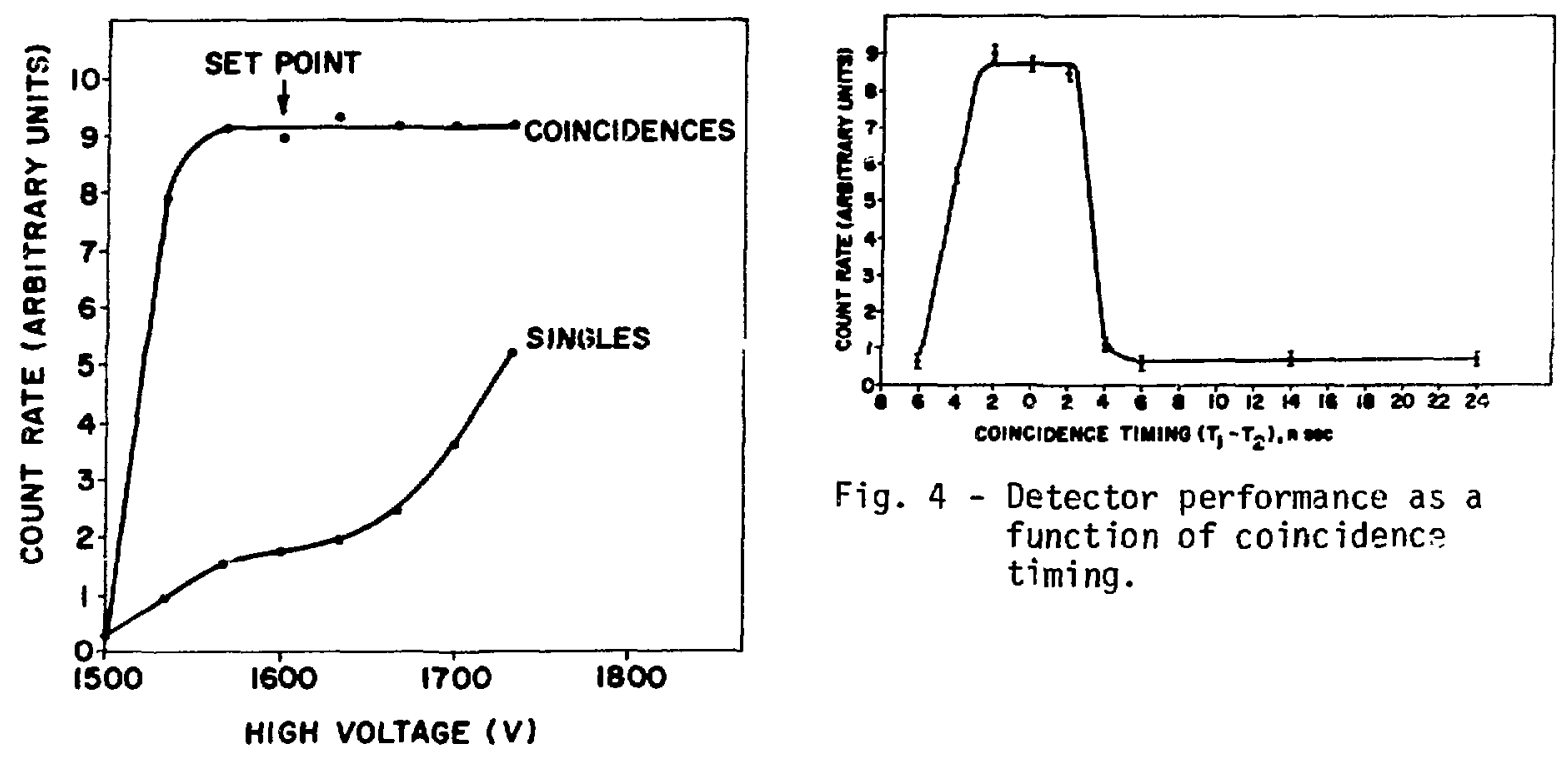

Fig. 4 - Detector performance as a function of coincidenc? timing.

Fig. 3 - Detector performance as a function of phototube HV.

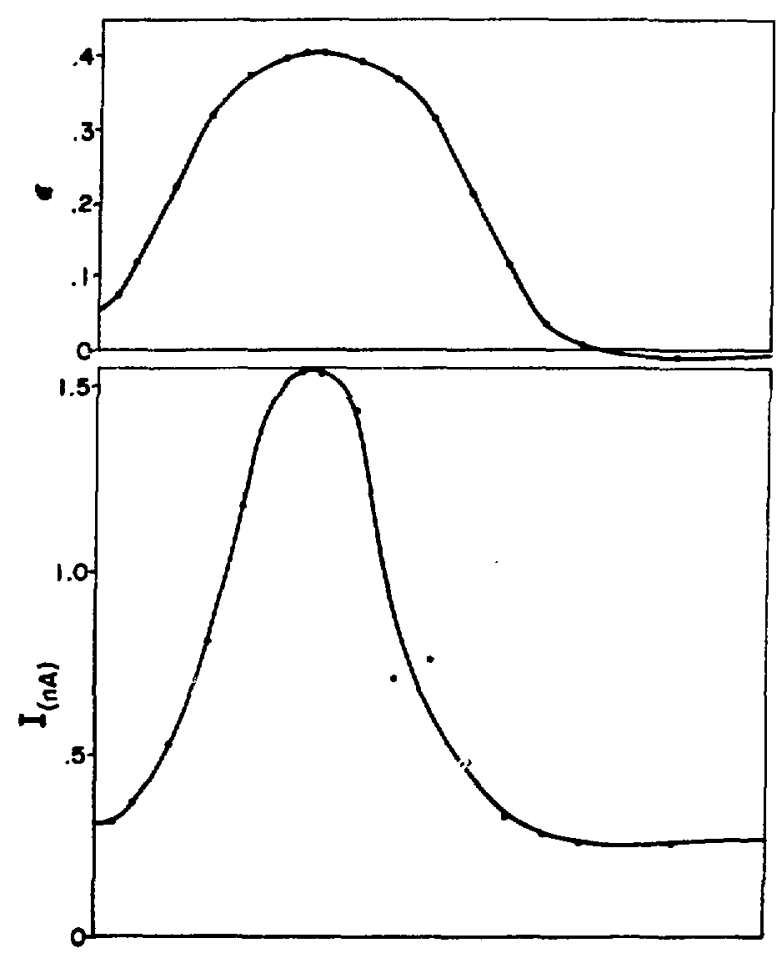

8 (ARBITAARY UNITS;

Fig. 5 - Asymmetry $\varepsilon$ and intensity I as a function of spin filter magnetic field (solenoid current). This illustrates the $\Delta B$ quench. 
TABLE I - POLARIMETER GEOMETRY. [Width, etc., refer to horizonta] detectors $(L-R)$; the U-D detectors are identical.]

\begin{tabular}{|c|c|c|c|c|c|c|c|c|c|c|}
\hline \multirow[b]{2}{*}{ Polarimeter } & \multirow{2}{*}{$\begin{array}{l}\text { Arigle } \\
\text { (deg) } \\
\end{array}$} & \multirow{2}{*}{$\begin{array}{l}\text { Radius } \\
\text { (inch) } \\
\end{array}$} & \multirow{2}{*}{$\begin{array}{l}\text { Width } \\
\text { (inch) }\end{array}$} & \multirow{2}{*}{$\begin{array}{l}\text { Height } \\
\text { (inch) } \\
\end{array}$} & \multirow{2}{*}{$\begin{array}{l}\text { Thick } \\
\text { (inch) }\end{array}$} & \multirow{2}{*}{$\begin{array}{l}\text { Typical } \\
\text { Target } \\
\text { (inch) }\end{array}$} & \multicolumn{2}{|c|}{ Rates/pc } & \multicolumn{2}{|c|}{$\begin{array}{l}\text { Max. Beam Spot } \\
(\mathrm{rms}, \mathrm{mm})\end{array}$} \\
\hline & & & & & & & Singles & Coincidence & Width & $\mathrm{He}$ ight \\
\hline$E P B$ & 17.0 & 18.75 & .75 & .75 & .50 & $2 \times 2 \times .01$ & 10 & 1 & 10 & 5 \\
\hline EPB & $66.4^{7}$ & 6.8 & .75 & 1.625 & .25 & $2 \times 2 \times .01$ & 20 & 1 & & \\
\hline $\mathrm{LB}^{3}$ & 17.0 & 21.4 & .75 & .75 & .50 & $2 \times 2 \times .002$ & 1 & .1 & 20 & 10 \\
\hline$L B^{3}$ & 68.0 & 6.75 & 1.0 & 3.0 & .25 & $2 \times 2 \times .002$ & 5 & .1 & & \\
\hline
\end{tabular}

1 This angle should be changed for low energy operation.

2 Maximum target thickness $=0.25$ inch.

3 Note that the $16^{\circ}$ bend (LB-BM-05) between the polarimeter and $L D_{2}$ targets precesses horizontal spin components through $53^{\circ}$ at $800 \mathrm{MeV}$. 
TABLE II - POLARIMETER DATA

\begin{tabular}{lccc}
\hline Reaction & $\begin{array}{c}\text { Angle } \\
(1 \mathrm{ab})\end{array}$ & $\underline{\text { Energy }}$ & Analyzing Power \\
\hline EPB Polarimeter & 17.0 & 796 & $.481 \pm .002$ \\
EPB Polarimeter & 17.0 & 787 & $.486 \pm .003$ \\
EPB Polarimeter & 17.0 & 643 & $.538 \pm .002$ \\
EPB Polarimeter & 17.0 & 428 & $.465 \pm .010$ \\
LB Polarimeter & 17.0 & 800 & $.46 \pm .01$ \\
pp $\rightarrow$ pp & 16.9 & 796 & $.495 \pm .003$ \\
pp $\rightarrow$ pp & 16.9 & 787 & $.501 \pm .003$ \\
pp $\rightarrow$ pp & 17.4 & 643 & $.548 \pm .004$ \\
pp $\rightarrow$ pp & 17.0 & 428 & $.476 \pm .011$ \\
pp $\rightarrow$ pp & 24.0 & 428 & $.387 \pm .008$ \\
\hline \hline
\end{tabular}

